\title{
Coberturas vacinais superestimadas? Novas evidências a partir do inquérito de Pau da Lima' ${ }^{1}$
}

\author{
Ligia Maria Vieira da Silva, ${ }^{2}$ Vera Lúcia Formigli, ${ }^{3}$ \\ Macius Pontes Cerqueira ${ }^{4}$ e Leonardo Kruchevsky ${ }^{4}$
}

RESUMO Com o objetivo de avaliar a cobertura vacinal em crianças de 0 a 5 anos, um estudo transversal com base em inquérito domiciliar foi realizado em 1992 no Distrito Sanitário de Pau da Lima, Salvador, Bahia, Brasil, através do recurso à técnica de amostragem por conglomerados. Foram sorteadas 30 microáreas homogêneas no que diz respeito às características socioeconômicas. Informações sobre o estado vacinal de 385 crianças foram obtidas a partir da verificação do cartão de vacinação e da senha de campanha, ou, na ausência destes, a partir da informação verbal da mãe ou responsável. A cobertura vacinal encontrada foi de $70 \%$ para a vacina antipólio; de $56 \%$ para a vacina tríplice; de $74 \%$ para a vacina anti-sarampo; e de $87 \%$ para a BCG, considerando-se as diversas fontes de informação em todas as faixas etárias. Estes resultados indicam uma possível superestimação das taxas obtidas a partir de registros rotineiros dos serviços de saúde e das campanhas. Os autores discutem as implicações das baixas coberturas encontradas em todas as faixas etárias e, mais acentuadamente, entre menores de 1 ano.

As doenças imunopreveníveis ainda são um problema de saúde da infância nos países periféricos. Segundo estimativas do Fundo das Nações Unidas para a Infância (UNICEF), o sarampo, o tétano e a coqueluche estão entre as doenças que mais matam menores de

1 Trabalho realizado com o auxílio do Conselho Nacional de Desenvolvimento Científico e Tecnológico (CNPq) (processo CNPq n 500246/91-2). Apresentado no III Congresso Brasileiro de Epidemiologia, Salvador, Bahia, 24 a 28 de abril de 1995.

2 Instituto de Saúde Coletiva da Universidade Federal da Bahia. Rua Padre Feijó, 29, $4^{\circ}$ andar, CHR, Canela, CEP 40110-170, Salvador, BA, Brasil. Correspondência e pedidos de separatas devem ser enviados para este endereço.

3 Departamento de Medicina Preventiva da Universidade Federal da Bahia, Salvador, BA, Brasil.

4 Bolsistas de iniciação científica do CNPq, Instituto de Saúde Coletiva da Universidade Federal da Bahia, Salvador, BA, Brasil.
5 anos. Em 1992, essas doenças foram responsáveis por cerca de dois milhões de mortes em países em desenvolvimento (1). A elevação da cobertura vacinal dessas enfermidades de um patamar entre 20 e 35\% em 1984 para 75 e $90 \%$ em 1992 tem sido associada, entre outros fatores, à redução da mortalidade infantil verificada neste período (1). A incidência da poliomielite reduziu-se significativamente no mundo e, nas Américas em particular, o último caso relacionado com um vírus autóctone selvagem ocorreu no Peru em 1991 (2).

No Brasil, a adoção da estratégia de campanhas nacionais para a imunização contra a poliomielite resultou na elevação das coberturas vacinais de 89\% em 1993 para 97\% em 1994 (3). Também as coberturas de rotina para as vacinas tríplice (DPT), anti-sarampo (AS) e Bacilo de Calmet e Guérin (BCG) foram ampliadas ao longo da década de 80 (4).

No estado da Bahia, a situação é diferenciada tendo em vista que não apenas as coberturas de rotina (4) são baixas, mas também as coberturas decorrentes da realização das campanhas situam-se em patamares insuficientes para o controle das respectivas doenças. Entre 1991 e 1993 verificou-se uma redução da cobertura vacinal decorrente da campanha antipólio para crianças menores de 1 ano, de 83\% em 1991 (5) para 76\% em 1993 (6).

Na cidade de Salvador, em 1991, as coberturas de rotina para as vacinas DPT, AS e BCG em crianças menores de 1 ano foram de 47, 65 e 76\%, respectivamente (5). A cobertura da vacina 
antipólio (APO), segundo dados de campanha, foi elevada de 65\% em 1991 para 74\% em 1992 (6), reduzindo-se em 1993 para 67\% (6). Este último percentual é inferior àquele considerado pela Comissão Internacional para a Certificação da Erradicação da Poliomielite (CICEP) como necessário para manter a erradicação da pólio.

O cálculo da cobertura vacinal a partir dos dados de rotina pode incorrer em erros, principalmente devido às dificuldades existentes para a realização de estimativas populacionais precisas nos anos subseqüentes aos censos. A não-utilização dos serviços pela população moradora na área de abrangência da unidade sanitária ou a utilização por parte de pessoas residentes em outras áreas geográficas também podem interferir com o cálculo da cobertura real. As estimativas realizadas a partir do número total de doses aferidas nas campanhas, por sua vez, podem não corresponder à cobertura real, tendo em vista que não há informação sobre cada criança individualmente. Em ambas as situações, tanto uma subestimação como uma superestimação dos parâmetros da cobertura "verdadeira" podem ocorrer.

Com o objetivo de suprir a ausência e deficiência da informação sobre a cobertura vacinal decorrente de dados oriundos dos registros rotineiros de saúde, o Programa Ampliado de Imunizações (PAI) da Organização Mundial da Saúde (OMS) recomenda desde 1978 a realização de inquéritos domiciliares para avaliação da cobertura vacinal $(7,8)$.

O presente estudo faz parte de um projeto mais amplo que visa a desenvolver estratégias para a avaliação global de sistemas locais de saúde (9). A cobertura assistencial e a cobertura específica por programas e ações desenvolvidas constituem-se em atributos de um sistema de saúde relevantes para avaliação. Por esses motivos, o objetivo da investigação aqui descrita foi estimar a cobertura para as vacinas APO, DPT, AS e BCG no Distrito Sanitário de Pau da Lima. No mesmo estudo foram investigadas também as características da utilização dos serviços de saúde do Distrito Sanitário de
Pau da Lima, que foram objeto de publicação anterior (10).

\section{MATERIAIS E MÉTODOS}

Foi realizado um estudo transversal conduzido com base em inquérito domiciliar no Distrito Sanitário de Pau da Lima, situado no Município de Salvador, Bahia, Brasil. A população do referido distrito em 1991 era de 143688 habitantes, segundo dados do censo demográfico realizado pelo Instituto Brasileiro de Geografia e Estatística (IBGE), sendo que 16591 estavam na faixa etária entre 0 e 4 anos. Tendo em vista que para os estratos considerados no estudo em relação à idade não havia informação censitária disponível, foi feita uma estimativa baseada na distribuição da população por faixa etária para Salvador (11).

Foi utilizada uma técnica de amostragem por conglomerados recomendada pela OMS através do PAI para a aferição de cobertura vacinal $(7,8)$ diante das facilidades relacionadas com sua operacionalização. Optou-se pela definição de um erro de 0,05 menor do que o erro proposto pelo PAI, de 0,10 - resultando em um tamanho amostral de 384 indivíduos, determinado de acordo com Lwanga e Lemeshow (12). A escolha da amostra de tamanho maior do que aquela recomendada pelo PAI (= 210) decorreu da realização concomitante de estudo de utilização de serviços de saúde, evento menos freqüente do que a vacinação (10).

Foram sorteadas 30 microáreas dentre as 50 identificadas pela gerência do Distrito a partir das características socioeconômicas tais como renda, tipo de habitação, tipo de abastecimento de água e tipo de esgoto sanitário (13). Em cada microárea foi sorteada uma rua, na qual foram colhidas informações acerca da cobertura vacinal de 13 crianças na faixa etária entre 0 e 5 anos, moradoras de domicílios contíguos, visitados em seqüência, a partir do número mais baixo. Nas microáreas referentes a favelas ou invasões, onde não havia ruas delimitadas, iniciou-se pelo primeiro domicílio encon- trado, continuando-se as entrevistas nos domicílios subseqüentes até completar 13 crianças. As informações foram prestadas pela mãe da criança, ou, na sua ausência, por membro da família presente no domicílio no momento da entrevista. As entrevistas foram realizadas por estudantes de Medicina, bolsistas do $\mathrm{CNPq}$ e por médicos residentes vinculados ao programa de residência em Medicina Social do Instituto de Saúde Coletiva da Universidade Federal da Bahia, treinados com esta finalidade. Em outubro de 1992 foi realizado pré-teste envolvendo cerca de 15 domicílios, localizados no Distrito, com a finalidade de analisar a adequação do questionário. A coleta de dados realizou-se nos meses de novembro a dezembro de 1992. Das 390 crianças entrevistadas, cinco casos foram descartados devido à ausência de informação sobre a idade.

O número de doses tomado por cada criança foi anotado a partir do registro das doses no cartão fornecido pelas unidades de saúde para as vacinas APO, AS, DPT e BCG. Quando o entrevistado não dispunha de cartão, o entrevistador anotava o número de doses tomados por vacina segundo a informação verbal. $\mathrm{O}$ mesmo procedimento foi adotado para a vacinação das campanhas (número de doses registradas no cartão ou informação verbal). Com esta finalidade foi utilizado o questionário elaborado por Silva e Mota em 1986 (14). Os dados foram digitados, consolidados e analisados no programa EPI-INFO.

Foi considerada como imunizada a criança que havia tomado o número de doses adequado para a sua idade: três ou mais doses para APO e DPT em maiores de 1 ano, uma dose para AS em maiores de 1 ano e uma dose para BCG em qualquer idade. Foi considerada apenas uma dose para o sarampo tendo em vista que a adoção da norma de vacinação que preconiza duas doses ainda estava em fase de implementação no Município. As crianças menores de 6 meses que estavam cumprindo com o calendário vacinal foram consideradas "cobertas" (por exemplo, uma criança de 2 meses com uma dose 
de APO, DPT e BCG). Foram calculadas as proporções de crianças consideradas imunizadas segundo esses critérios para todas as fontes de informação (cartão, informação verbal referente à rotina, senha de campanha e informação verbal referente à campanha). O cálculo da cobertura total foi feito a partir da intersecção entre as diversas fontes de informação. O intervalo de confiança para a proporção de crianças com o esquema vacinal completo foi estimado a partir da fórmula $p \pm \mathrm{Zc} \sqrt{p q / \mathrm{N}}$, conforme Spiegel (15).

\section{RESULTADOS}

Como já foi mencionado, das 390 crianças entrevistadas, cinco casos foram descartados devido à ausência de informação sobre a idade. Dentre as 385 crianças remanescentes, 72 (19\%) eram menores de 1 ano, 73 (19\%) estavam na faixa entre 12 e 23 meses, 161 $(42 \%)$ estavam na faixa entre 24 e 48 meses e 79 (20\%) eram maiores de 48 meses. A comparação entre a distribuição da população segundo faixa etária na amostra e na população do Distrito Sanitário de Pau da Lima revela diferenças discretas entre os menores de 1 ano ( $2 \%$ ) e no grupo de 24 a 48 meses (4\%). Nos 385 casos estudados havia algum tipo de informação vacinal: registro em caderneta, registro em senha, informação verbal sobre vacinação de rotina ou informação verbal sobre campanha.

Chama a atenção a existência de crianças que não haviam tomado nenhuma dose de vacina, em proporções que variaram entre $4 \%$ (BCG) e $25 \%$

TABELA 1. Vacinação de rotina segundo tipo de vacina e número de doses registradas em caderneta, Distrito Sanitário de Pau da Lima, Salvador, 1992

\begin{tabular}{|c|c|c|c|c|c|c|c|c|}
\hline \multirow[b]{2}{*}{ Número de doses } & \multicolumn{2}{|c|}{ APO } & \multicolumn{2}{|c|}{ DPT } & \multicolumn{2}{|c|}{ AS } & \multicolumn{2}{|c|}{ BCG } \\
\hline & No. & $\%$ & No. & $\%$ & No. & $\%$ & No. & $\%$ \\
\hline 0 & 62 & 16 & 56 & 14 & 97 & 25 & 16 & 4 \\
\hline 1 & 40 & 10 & 32 & 8 & 147 & 38 & 218 & 57 \\
\hline 2 & 47 & 12 & 42 & 11 & 13 & 3 & 2 & 0 \\
\hline 3 & 68 & 18 & 77 & 20 & - & - & - & - \\
\hline+4 & 57 & 15 & 68 & 18 & - & - & - & - \\
\hline Sem caderneta & 111 & 29 & 110 & 29 & 128 & 34 & 149 & 39 \\
\hline Total & 385 & 100 & 385 & 100 & 385 & 100 & 385 & 100 \\
\hline
\end{tabular}

(AS), como mostra a tabela 1 . Um número razoável de crianças $(29 \%)$ não possuía cartão no momento da entrevista. Tendo em vista que entre as últimas apenas $10 \%$ não haviam sido vacinadas, segundo a informação verbal do responsável, é possível que o cartão tenha sido perdido.

Observa-se uma progressiva elevação da cobertura vacinal com a idade para todos os tipos de vacina (tabelas 2-5), o que corresponde a dizer que o grupo mais descoberto é aquele de menores de 1 ano, precisamente $\mathrm{o}$ mais vulnerável. A menor cobertura vacinal foi observada na faixa etária de 0 a 12 meses para a vacina DPT $(28 \%)$, como mostra a tabela 3 . A maior cobertura, de $96 \%$, correspondeu à vacina BCG na faixa etária acima de 48 meses. Entre os menores de 1 ano, as coberturas para todas as vacinas estão abaixo de $80 \%$, sendo que no caso da APO e DPT elas são inferiores a 50\%. Os melhores percentuais obtidos pela AS não são indicativos de cobertura adequada, tendo em vista que apenas uma dose desta vacina não é suficiente para imunizar todas as crianças, principalmente as menores de 1 ano (16).

Os intervalos de confiança de $95 \%$ estimados para as coberturas totais revelaram pequena variação em relação às proporções encontradas: APO (65-75); DPT (51-61); AS (70-79); e BCG (84-90), como mostra a tabela 6.

As razões alegadas para a vacinação estar incompleta foram relacionadas com crenças, tabus e mesmo com o desconhecimento acerca da oferta do serviço de saúde (itens 5, 8, 10, 15-19 da tabela 7), que correspondem a $17 \%$. Por fim, somando $16 \%$ do conjunto, estão os motivos relacionados com deficiências dos serviços de saúde (itens 6, 7, 9 e 13 da tabela 7), além de razões relacionadas com possível negligência da mãe, como "esqueceu de levar a criança" (8\%).

\section{DISCUSSÃO E CONCLUSÕES}

As coberturas vacinais encontradas para o Distrito Sanitário de Pau da

TABELA 2. Cobertura vacinal antipólio (APO) de rotina e em campanha de vacinação segundo idade e fonte de informação, Distrito Sanitário de Pau da Lima, Salvador, 1992

\begin{tabular}{|c|c|c|c|c|c|c|c|c|c|c|c|}
\hline \multirow[b]{2}{*}{$\begin{array}{l}\text { Idade } \\
\text { (meses) }\end{array}$} & \multicolumn{4}{|c|}{ Vacinação de rotina } & \multicolumn{4}{|c|}{ Campanha de vacinação } & \multicolumn{2}{|c|}{ Cobertura total } & \multirow[b]{2}{*}{ Tota } \\
\hline & Caderneta & $\%$ & $\begin{array}{c}\text { Informação verbal } \\
\text { e caderneta }\end{array}$ & $\%$ & $\begin{array}{l}\text { Senha da } \\
\text { campanha }\end{array}$ & $\%$ & $\begin{array}{c}\text { Informação } \\
\text { verbal e senha }\end{array}$ & $\%$ & No. & $\%$ & \\
\hline $12-23$ & 28 & 38 & 38 & 52 & 24 & 33 & 34 & 47 & 49 & 67 & 73 \\
\hline $23-48$ & 53 & 33 & 91 & 56 & 61 & 38 & 91 & 56 & 125 & 78 & 161 \\
\hline$>48$ & 32 & 40 & 48 & 61 & 36 & 46 & 49 & 62 & 68 & 86 & 79 \\
\hline
\end{tabular}


TABELA 3. Cobertura vacinal tríplice (DPT) de rotina e em campanha de vacinação segundo idade e fonte de informação, Distrito Sanitário de Pau da Lima, Salvador, 1992

\begin{tabular}{|c|c|c|c|c|c|c|c|c|c|c|c|}
\hline \multirow[b]{2}{*}{$\begin{array}{l}\text { Idade } \\
\text { (meses) }\end{array}$} & \multicolumn{4}{|c|}{ Vacinação de rotina } & \multicolumn{4}{|c|}{ Campanha de vacinação } & \multicolumn{2}{|c|}{ Cobertura total } & \multirow[b]{2}{*}{ Total } \\
\hline & Caderneta & $\%$ & $\begin{array}{c}\text { Informação verbal } \\
\text { e caderneta }\end{array}$ & $\%$ & $\begin{array}{l}\text { Senha da } \\
\text { campanha }\end{array}$ & $\%$ & $\begin{array}{c}\text { Informação } \\
\text { verbal e senha }\end{array}$ & $\%$ & No. & $\%$ & \\
\hline $12-23$ & 30 & 41 & 38 & 52 & 4 & 5 & 4 & 5 & 38 & 52 & 73 \\
\hline $23-48$ & 61 & 38 & 95 & 59 & 3 & 2 & 11 & 7 & 97 & 60 & 161 \\
\hline$>48$ & 43 & 54 & 61 & 77 & 2 & 2 & 7 & 9 & 61 & 77 & 79 \\
\hline
\end{tabular}

TABELA 4. Cobertura vacinal anti-sarampo (AS) de rotina e em campanha de vacinação segundo idade e fonte de informação, Distrito Sanitário de Pau da Lima, Salvador, 1992

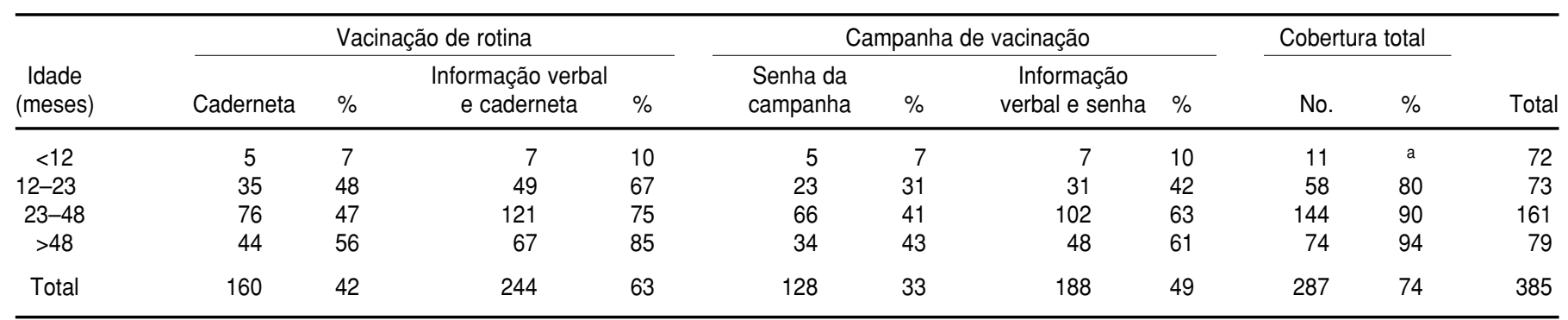

a Não foi calculada cobertura vacinal para essa faixa etária.

TABELA 5. Cobertura vacinal de rotina contra tuberculose (BCG) e presença de cicatriz segundo idade e fonte de informação, Distrito Sanitário de Pau da Lima, Salvador, 1992

\begin{tabular}{|c|c|c|c|c|c|c|c|c|c|}
\hline \multirow[b]{2}{*}{$\begin{array}{l}\text { Idade } \\
\text { (meses) }\end{array}$} & \multicolumn{4}{|c|}{ Vacinação de rotina } & \multicolumn{2}{|c|}{ Cicatriz } & \multicolumn{2}{|c|}{ Cobertura total } & \multirow[b]{2}{*}{ Total } \\
\hline & Caderneta & $\%$ & $\begin{array}{c}\text { Informação verbal } \\
\text { e caderneta }\end{array}$ & $\%$ & Presente & $\%$ & No. & $\%$ & \\
\hline $12-23$ & 42 & 57 & 57 & 78 & 44 & 60 & 59 & 81 & 73 \\
\hline $23-48$ & 87 & 54 & 138 & 86 & 118 & 73 & 145 & 90 & 161 \\
\hline$>48$ & 51 & 65 & 75 & 95 & 61 & 77 & 76 & 96 & 79 \\
\hline
\end{tabular}

Lima são inferiores àquelas obtidas através dos registros rotineiros das unidades de saúde e das campanhas. Para esse Distrito, as coberturas calculadas a partir dos registros das doses aplicadas nas três etapas da campanha antipólio de 1992 para crianças de 0 a 4 anos foram, respectivamente, $80,75 \mathrm{e}$ $76 \% .^{5}$

Em 1986, inquérito realizado por Silva e Mota evidenciou 89\% de cober-

\footnotetext{
5 Distrito Sanitário de Pau da Lima (DSPL). Relatório de 1992 [documento mimeografado]. Salvador: Gerência do DSPL; 1993.
}

tura vacinal antipólio em crianças na faixa de 1 ano de idade e $80 \%$ para a faixa de 7 a 11 meses de residentes em áreas urbanas do Estado da Bahia, sendo que para a região metropolitana de Salvador o percentual de crianças na faixa de 1 ano com três doses de APO foi de 91,0\% considerando-se todas as informações disponíveis (14). Um segundo trabalho, realizado pela Secretaria Estadual de Saúde em Salvador nos meses de setembro a dezembro de 1992 estimou a cobertura vacinal de crianças residentes em Salvador em $69 \%$ para DPT (três ou mais doses); $96 \%$ para APO (três ou mais doses); 92\% para AS (uma ou mais doses); e $88 \%$ para BCG (uma dose) (17). Com exceção da vacina BCG, os percentuais encontrados divergem dos resultados do presente estudo quando comparadas as mesmas faixas etárias. Em contrapartida, um outro estudo realizado em 1993 pela Secretaria Municipal de Saúde para Salvador encontrou coberturas vacinais confirmadas de $47 \%$ para a vacina APO (três doses); $46 \%$ para a vacina DPT (três doses) $45 \%$ para a AS e $90 \%$ para a BCG. Quando as informações verbais 
TABELA 6. Cobertura vacinal total para crianças de 0 a 5 anos segundo tipo de vacina e estimativa de intervalo de confiança (IC), Distrito Sanitário de Pau da Lima, Salvador, 1992

\begin{tabular}{ccc}
\hline Tipo de vacina & $\begin{array}{c}\text { Cobertura (\%) } \\
n^{\mathrm{a}}=385\end{array}$ & IC95\% \\
\hline APO & 70 & $65-75$ \\
DPT & 56 & $51-61$ \\
AS & 74 & $70-79$ \\
BCG & 87 & $84-90$ \\
\hline
\end{tabular}

a Total de crianças.

foram consideradas, essas coberturas subiram para 70, 66, 65 e 91\%, respectivamente (18). A comparação entre o trabalho de Silva e Mota (14) e aquele realizado pela Secretaria Municipal de Saúde em 1993, cujo maior percentual para as vacinas APO, DPT e AS foi de $70 \%$ na faixa etária entre 12 e 23 meses, revela um decréscimo da cobertura vacinal segundo critério proposto por Henderson e Sundaresan para comparação entre dois inquéritos (7).

Embora os estudos acima mencionados tenham utilizado amostragem por conglomerados conforme recomendação do PAI (7), a metodologia variou no que diz respeito ao cálculo do tamanho da amostra, ao conglomerado escolhido e às faixas etárias analisadas. O inquérito realizado por Silva e Mota em 1986 (14) envolveu 4116 crianças da região metropolitana de Salvador distribuídas por nove faixas etárias: 0, 2, 3, 4, 5, 6 meses; 7 a 11 meses; 1 ano; e 2 a 4 anos. No outro estudo referido, também desenvolvido pela Secretaria Estadual de Saúde em 1992 (17), a cobertura vacinal foi examinada em apenas 46 crianças do Município de Salvador, na faixa etária entre 12 e 23 meses. Por sua vez, a investigação conduzida pela Secretaria Municipal de Saúde de Salvador em 1993 empregou uma amostra de 858 crianças na faixa etária entre 12 e 23 meses (18). Já no presente trabalho, a cobertura vacinal foi pesquisada em um distrito sanitário de Salvador entre 385 crianças distribuídas por quatro faixas etárias: menos de 12 meses; entre 12 e 23 meses; entre 24 e 48 meses; e mais de 48 meses de idade. No que diz respeito à unidade de referência considerada como conglomerado, também houve variação entre os estudos analisados: o primeiro estudo (14) utilizou o distrito municipal; as

TABELA 7. Razões alegadas para a vacinação incompleta, Distrito de Pau da Lima, Salvador, 1992

\begin{tabular}{lrr}
\hline \multicolumn{1}{c}{ Razões } & No. & $\%$ \\
\hline 1. Outros motivos & 33 & 20 \\
2. Não sabe & 27 & 16 \\
3. Não tem tempo de ir até a unidade de saúde & 26 & 15 \\
4. Esqueceu de levar a criança & 14 & 8 \\
5. A criança está sempre doente & 10 & 6 \\
6. Não existem vacinas na unidade & 9 & 5 \\
7. A unidade de saúde não aplicou a vacina porque a criança estava doente & 8 & 5 \\
8. Acha que não existem outras vacinas ou outras doses & 8 & 5 \\
9. Não havia quem aplicasse vacina na unidade de saúde & 6 & 4 \\
10. A criança é muito nova & 6 & 4 \\
11. Sem informação & 4 & 2 \\
12. Não tem condições de levar a criança na unidade de saúde & 3 & 2 \\
13. Não era dia de vacina na unidade de saúde & 3 & 2 \\
14. Ignora que a vacina exista & 2 & 1 \\
15. A criança já teve a doença & 1 & 1 \\
16. A criança tem medo & 1 & 1 \\
17. Vacina provoca doença & 1 & 1 \\
18. Vacina não protege & 1 & 1 \\
19. Não sabe onde fazer vacinação & 1 & 1 \\
Total & 164 & 100 \\
\hline
\end{tabular}

duas outras investigações $(17,18)$ consideraram o setor censitário; e para o presente trabalho a unidade de referência foi a "microárea" homogênea do ponto de vista socioeconômico. Esses aspectos revelam graus de precisão distintos entre os quatro inquéritos, dificultando, porém não inviabilizando, a comparação entre os mesmos.

A hipótese das coberturas vacinais estarem de fato elevadas e de os dois inquéritos que registraram percentuais baixos - o presente inquérito e aquele conduzido por Porto et al. (18) - não terem captado essa realidade em decorrência de problemas de memória dos informantes, embora remota, deve ser considerada. A resposta a essa pergunta requer a validação da informação negativa através da verificação da proporção de soroconversão em sub-amostra das crianças cujas mães não possuíam nem cartão nem senha e que referiram não terem vacinado as crianças.

No entanto, alguns fatos falam a favor da validade da informação obtida através da presente investigação. Em primeiro lugar, as diferenças existentes entre o exame da cicatriz vacinal para BCG e as coberturas confirmadas e informadas dessa vacina foram pequenas, podendo-se afirmar existir concordância entre esses dois resultados (tabela 5). Por outro lado, o presente estudo e um dos inquéritos realizados em Salvador (18) têm resultados similares. Por fim, os próprios dados secundários da Secretaria Estadual de Saúde revelam uma tendência ao declínio da cobertura vacinal no Estado a partir de 1991, conforme mencionado anteriormente. Ou seja, existem fortes indicações de estar havendo superestimação das coberturas vacinais calculadas a partir das informações obtidas rotineiramente em Salvador e, em particular, em Pau da Lima.

As coberturas vacinais encontradas são insuficientes para o controle das doenças preveníveis pelas vacinas DPT, APO e AS. Esse fato revela-se na ocorrência de 91 casos de sarampo, 141 casos de coqueluche e 20 casos de difteria em Salvador em 1993, o que por si só já seria suficiente para determinar a 
adoção de medidas visando a aprimorar o monitoramento das coberturas vacinais. Isto suscita uma preocupação especial, tendo em vista a certificação da erradicação da poliomielite nas Américas em agosto de 1994 por parte da OMS/Organização Pan-Americana da Saúde (OPAS) (19). Embora a CICEP esteja convencida da interrupção da transmissão do poliovírus selvagem no continente, alerta para a possibilidade de reintrodução do mesmo em decorrência não só da sua circulação em outros países do mundo, como diante da existência de falhas em diversos programas. A CICEP chama atenção para a necessidade de se estender a cobertura vacinal além dos $80 \%$ atingidos pelos países nos últimos 6 anos, pois os poliovírus têm conseguido atingir suscetíveis em países com coberturas elevadas como o Canadá e os Países Baixos (9). A erradicação na Suécia foi possível com a imunização de $99 \%$ dos nascidos vivos na década de 40 (20). Nos Estados Unidos, Katz defendia a meta de ampliação da cobertura vacinal pela vacina oral contra a pólio para $100 \%$ dos menores de 2 anos, 10 anos após o controle da doença naquele país (21). Evidentemente, na vigência de coberturas inferiores a $80 \%$ esse risco deve aumentar consideravelmente. Por outro lado, o Brasil é um dos países que não desenvolvem todas as medidas preconizadas pela CICEP para a vigilância adequada da pólio (22). Até novembro de 1994, o Brasil não cumpria as metas de investigar o vírus nas fezes de pelo menos $80 \%$ dos indivíduos com paralisia flácida e de medir uma taxa mínima de
1/100 000 casos de paralisia flácida nos menores de 15 anos (22).

Diante desses fatos, é necessária uma atitude de cautela no que diz respeito à interpretação dos dados oficiais disponíveis. Uma superestimação das coberturas vacinais pode dificultar a definição de estratégias adequadas de controle para as doenças imunopreveníveis, havendo a possibilidade de que o país seja surpreendido por uma indesejável reintrodução do vírus da poliomielite ou pelo aumento do número de casos das doenças ainda não erradicadas.

Agradecimento. Os autores agradecem aos professores Vilma Santana e Naomar Almeida pelas críticas e sugestões.

\section{REFERÊNCIAS}

1. Fundo das Nações Unidas para a Infância (UNICEF). Situação mundial da infância. Brasília: UNICEF; 1994

2. Organización Panamericana de la Salud, Programa Especial de Salud Maternoinfantil y Población/Programa Ampliado de Inmunización. Boletín Informativo PAI No 5. Washington DC: OPAS; 1993.

3. Brasil, Ministério da Saúde, Fundação Nacional de Saúde, Programa Nacional de Imunizações. Série histórica de resultados das campanhas nacionais e do dia nordestino de vacinação com a vacina antipólio oral, Brasil 1980-1994. Brasília: Ministério da Saúde; 1995.

4. Brasil, Ministério da Saúde, Fundação Nacional de Saúde, Centro Nacional de Epidemiologia. Série histórica de coberturas vacinais de rotina por unidade federada, Brasil 1980-1991. Informe Epidemiológico do Sistema Único de Saúde 1992;5:141-148.

5. Bahia, Secretaria de Saúde do Estado da Bahia (SESAB), Centro de Informações de Saúde. Anuário estatístico: Informações de saúde - dados por município. Salvador: SESAB; 1993.

6. Bahia, Secretaria de Saúde do Estado da Bahia (SESAB), Centro de Informações de Saúde. Anuário estatístico: Informações de saúde - dados por município. Salvador: SESAB; 1994.

7. Henderson RH, Sundaresan T. Cluster sampling to assess immunization coverage: a review of experience with a simplified sampling method. Bull World Health Organ 1982; 60(2):253-260.

8. Lemeshow S, Robinson D. Surveys to measure programme coverage and impact: a review of the methodology used by the expanded programme on immunization. World Health Stat Q 1985;33:65-75.

9. Silva LMV, Formigli VLA. Avaliação e os projetos de reorganização das práticas de saúde: conceitos e abordagens. São Paulo: II Congresso Nacional da Rede IDA-Brasil; 1993.

10. Silva LMV, Formigli VLA, Cerqueira MP, Kruchevsky L, Teixeira MMA, Barbosa ASM et al. O processo de distritalização e a utilizacão de serviços de saúde: avaliação do caso de Pau da Lima. Cad Saude Publica 1995; 11(1):72-84.

11. Fundação Instituto Brasileiro de Geografia e Estatística (IBGE). Censo demográfico: resultados do universo relativos às características da população e dos domicílios, No 17, Bahia. Rio de Janeiro: IBGE; 1991.

12. Lwanga SK, Lemeshow S. Sample size determination in health studies. Geneva: World Health Organization; 1991.

13. Kadt E, Tasca R. Promovendo a eqüidade: um novo enfoque com base no setor da saúde. São Paulo-Salvador: Hucitec/Cooperação Italiana em Saúde; 1993.

14. Silva LMC, Mota ELA. Estudo por inquérito domiciliar da cobertura vacinal no estado da Bahia. Rev Baiana Saude Publica 1990;17(1/4): 45-79.

15. Spiegel MR. Estatística. $2^{a}$ ed. São Paulo: McGraw-Hill; 1985.

16. Aaby P, Pedersen IR, Knudsen K, Silva MC, Mordhorst CH, Helm Petersen NC et al. Child mortality related to seroconversion or lack of seroconversion after measles vaccination. Pediatr Infect Dis J 1989;8:197-200.
17. Bahia, Secretaria de Saúde do Estado da Bahia (SESAB), Universidade Federal de Pelotas (UFP), Fundo das Nações Unidas para a Infância (UNICEF). Diagnóstico de saúde das crianças e mulheres da Bahia. Salvador: SESAB/ UFP/UNICEF; 1993.

18. Porto LA, Santos MSF, Santos SDP, Araújo EC, Melo C, Vilasbôas ALQ. Cobertura vacinal em Salvador. Olinda: IV Congresso Brasileiro de Saúde Coletiva - Associação Brasileira de Pós-Graduação em Saúde Coletiva (ABRASCO); 1994

19. Organización Panamericana de la Salud, Programa Especial de Salud Maternoinfantil y Población/Programa Ampliado de Imunización. Boletín Informativo PAI No 4. Washington DC: OPAS; 1994.

20. Bottiger MA. A study of the seroimmunity that has protected the Swedish population against poliomyelitis for 25 years. Scand J Infect Dis 1987;19:595-601.

21. Katz SL. Poliovirus vaccine policy. Am J Dis Child 1989;143:1007-1009.

22. Organización Panamericana de la Salud, Programa Especial de Salud Maternoinfantil y Población/Programa Ampliado de Inmunización. Boletín Informativo PAI No 6. Washington DC: OPAS; 1994.

Manuscrito recebido em 6 de setembro de 1995. Versão revisada aceita para publicação em 24 de junho de 1996. 
ABSTRACT To evaluate vaccination coverage in children 0 to 5 years of age, a cross-sectional study based on a household survey was carried out in 1992 in the Pau de Lima Health District, Salvador, Bahia, Brazil, using a cluster sampling technique. The district was subdivided into 30 small areas that were homogeneous with respect to socioeconomic characteristics. Information on the vaccination status of 385 children was obtained through verification of a vaccination card or campaign voucher, or, in the absence of these items, through verbal confirmation from the mother or other responsible person. Based on all the sources of information, the study found vaccination coverage rates in the entire age range of $69 \%$ for polio vaccine; $56 \%$ for DTP; $74 \%$ for measles vaccine; and $87 \%$ for $B C G$. These results suggest that rates obtained from routine records of the health services and vaccination campaigns may be overestimates. The authors discuss the implications of the low coverage rates found in the entire age group and especially among children under 1 year old.

\section{PROGRAMA DE BECAS DEL INSTITUTO MEMORIAL GORGAS}

Fecha límite para solicitudes: junio de 1998

Antecedentes: El Instituto Memorial Gorgas de Medicina Tropical y Preventiva (GMI) fundado por el General William Crawford Gorgas ha apoyado por largo tiempo la investigación y el control de las enfermedades en Panamá y Centroamérica. Desde su reubicación en el Centro William Gorgas para la Medicina Geográfica en la Universidad de Alabama en Birmingham, el GMI ha continuado apoyando la investigación y la capacitación de personal para el control de las enfermedades tropicales. Con el fin de fomentar enlaces científicos entre Panamá, otros países de Centroamérica y las naciones del Caribe con los Estados Unidos de América, el GMI patrocina becas para apoyar a corto plazo a investigadores jóvenes de la Región, de manera que: 1) colaboren en investigaciones biomédicas sobre enfermedades tropicales de importancia para la salud pública de esas zonas, (2) aprendan técnicas y métodos nuevos que puedan aplicarse al estudio de esos padecimientos, establezcan lazos de comunicación científica, e inicien programas cooperativos de estudios y de investigación.

Elegibilidad: Los candidatos deberán tener el nivel educativo y conocimientos de su especialidad correspondientes a un doctorado o tener un título de médico. Pueden hacerse excepciones para candidatos con experiencia y conocimientos excepcionales. Deben ser ciudadanos o residentes permanentes de Panamá, Estados Unidos 0 algún país de Centroamérica 0 el Caribe, y desempeñar un puesto de investigador o médico en una institución de salud pública o de enseñanza en su país. Su participación en este programa deberá ser avalada por la institución a la que presta sus servicios. Los solicitantes a esta beca deberán tener menos de 40 años de edad, aunque en casos excepcionales podrán considerarse candidatos mayores.

Financiamiento: Las becas son otorgadas y administradas por la Sociedad Americana de Medicina Tropical e Higiene. Por lo general no exceden de US\$10 000 para cubrir el viaje de ida y vuelta, alojamiento y materiales requeridos para el proyecto, el cual no se extenderá por más de 3 meses. El número de becas depende de la disponibilidad de fondos, pero la intención del programa es ofrecer por lo menos dos becas anuales. Cada beca será entregada directamente al solicitante, quien responderá por ella a la Secretaría del Comité de Becas de la Sociedad Americana de Medicina Tropical e Higiene.

Procedimiento de solicitud: Antes de junio de 1998 deberá enviarse lo siguiente:

1) Propuesta de no más de tres páginas escritas a máquina, en la que se describan las metas del proyecto, los métodos o plan de acción, y su importancia en relación con los problemas de salud de Centroamérica y Panamá.

2) Un resumen personal del solicitante y copias de no más de cinco publicaciones recientes escogidas según su relevancia para el proyecto o su importancia en este campo.

3) Una carta de un funcionario autorizado de la institución a que esté afiliado el solicitante, con el fin de verificar que el proyecto y el período de viaje se considerarán asunto oficial.

4) Una carta del investigador de la institución anfitriona, en la que indique su interés en colaborar en el proyecto y describa su participación y cualquier instalación especial que ofrezca, así como también un resumen personal del propio investigador.

5) Documentos que corroboren que el proyecto ha sido revisado y aprobado por los comités institucionales apropiados, si el proyecto involucra directamente a seres humanos 0 animales.

Para información y envío de solicitudes, escriba a:

Gorgas Memorial Institute Fellowship Program

American Society of Tropical Medicine and Hygiene

60 Revere Drive, Suite 500

Northbrook, IL 60062

Las solicitudes serán evaluadas por el Comité del GMI, que se reúne anualmente para seleccionar candidatos. Se tomarán en cuenta las cualidades del solicitante y de la institución anfitriona, la importancia regional del plan de investigación propuesto y una distribución geográfica equilibrada.

Una vez terminado el proyecto, cada becario debe presentar dentro de un plazo de 3 meses un informe escrito al Comité de Becas del GMI, que describa los logros alcanzados y rinda cuentas detalladas de la utilización de los fondos recibidos. Los únicos recibos que es obligatorio presentar son los del viaje de ida y vuelta. 